\title{
Vorticity in Green House Gases Flux Model in Boundary Layer
}

\author{
Valentini R. ${ }^{1}$, Vasenev I.I², Nurgaliev I.S.,** \\ ${ }^{1}$ Department of Forest Science and Environment, University of Tuscia, Via De Lellis, Viterbo, Italy \\ ${ }^{2}$ Laboratory Agro-ecological Monitoring, Russian State Agrarian University - Moscow Agricultural Academy named after \\ K.A.Timiryazev, Moscow, Russian \\ *Corresponding author: ildus58@mail.ru
}

Received July 25, 2013; Revised July 31, 2013; Accepted August 05, 2013

\begin{abstract}
Mathematical model of the turbulent flux in the three-layer boundary system is presented. Turbulence is described as a presence of the nonzero vorticity. Generalized advection-diffusion-reaction equation is derived for arbitrary number components in the flux. The fluxes in the layers are objects for matching requirements on the boundaries between the layers.
\end{abstract}

Keywords: green house gas, mathematical model, turbulent flux, vorticity, micrometeorology, material point, turbulent diffusion

Cite This Article: Valentini R., Vasenev I.I, and Nurgaliev I.S., "Vorticity in Green House Gases Flux Model in Boundary Layer." American Journal of Environmental Protection 1, no. 3 (2013): 66-69. doi: 10.12691/env-13-4.

\section{Introduction}

Modeling carbon dioxide and other green house gases (GHG) budgets at the local, regional and global scales is fundamental task in understanding the current and future behavior of the climate cycles and in working out strategies in decision making for sustainable development [1]. Mathematical models are effective tools and instruments, very often only possible, for verification emission reduction claims at different levels. The challenges in building adequate models always were large, especially in the presence of such complex phenomenon as turbulence [2]. Further complications are connected to the variability of fluxes in mixed landscapes, physical aspects in measuring covariance of fluxes and complex atmospheric layer dynamics as well as the problems of interpretation, gap filling, footprint identification, aggregation of data and others. Therefore, despite the fact that many investigators are producing defensible estimates of annual carbon exchange, it is imperative to clarify and to account all of the key constraints imposed to eddy covariance method by different factors. For example, well written just recently published book by Dr. George Burba [3], as well as his previous multiple publications, as an instrumental introduction to the subject and a thorough collection of useful state of the art articles [4] introduce simplified "Eddy Flux" as a product of the averaged air density and correlation term (time-averaged product) between fluctuations of the vertical air velocity and concentration fluctuations of the targeted gas component. The authors carefully perform necessary assessment of different sources of the errors. We try to use new platform to simulate the turbulent model and to account neglected component of the flow, such as vorticity induced turbulent diffusion in gas flux modeling (do not confuse with thermal diffusion: which is in English language literature called molecular diffusion though both types of diffusivity are molecular). Our approach is based on introducing vorticity and a new conception of the material point, constructed and effectively used by one of the authors in cosmology [5,6,7] and in modeling global social (demographic, economic) and natural (climatic) processes $[15,16]$.

\section{Task Formulation}

As usual, we represent system as simple as possible but not simpler as the epigraph calls. Atmosphere, canopy and soil are represented locally by flat horizontal layers (Figure 1). Dominated transport mechanisms identified on the Figure 1.

The fluxes between the layers have to fulfill the matching conditions as it is required in the well developed theory of partial differential equations. Fluxes' modeling encounters series of frontier complexities, one of which is incomplete knowledge on the nature of turbulence which is tackled in this work using vorticity conception. Eddy covariance method in its current state of the art serves as an available good temporary compromise answer to multiple open questions in the turbulent flux measurement and monitoring. Here we would emphasize on the negligence of the two diffusive fluxes in the main eddy covariance expression: molecular diffusion and turbulent diffusion. Some authors suppose that developed turbulence mechanism covers all the aspects of the gas 
transport in the boundary layer. But, first of all, diffusive transport is main input/output of gases and, therefore, has to be counted and estimated separately in the layer flux balance. And, secondly, if we plan to build self-consistent models of the gas circulation within all three subsystems, diffusion is the main phenomenon in the soil layer.
Therefore, in the traditional expression of eddy covariance flux, speed of air has to be replaces by speed of considered gas. Therefore the problem of instrumental discrimination between speed of air and, say, carbon dioxide arises. Some other constraints of the method are discussed by Baldocchi D.D. [8].

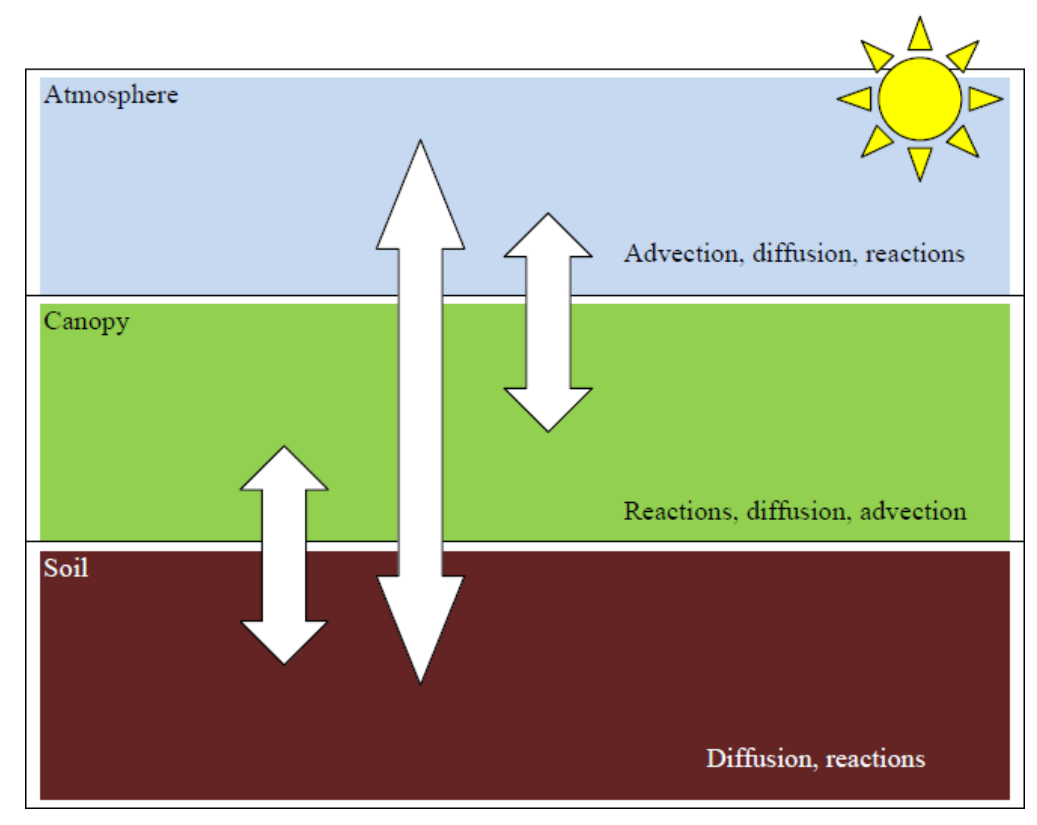

Figure 1. The components of the model

\section{GHG Flux Model Equations}

Typical sample formulae which are used in calculating eddy flux variables are following [9]

$$
\begin{gathered}
u_{*}=\overline{w^{\prime} U^{\prime}}=\frac{1}{N} \sum_{i=1}^{N} w^{\prime} U^{\prime} \\
\overline{w^{\prime} T^{\prime}}=\frac{1}{N} \sum_{i=1}^{N} w^{\prime} T^{\prime} \\
\overline{w^{\prime} x^{\prime}}=\frac{1}{N} \sum_{i=1}^{N}\left[\overline{w^{\prime} x^{\prime}}+\frac{m_{d} \rho_{X}}{m_{w} \rho_{d}} \overline{w^{\prime} \rho_{w}^{\prime}}+\rho_{X}\left(1+\frac{m_{d} \rho_{X}}{m_{w} \rho_{d}}\right) \frac{\overline{w^{\prime} T^{\prime}}}{T}\right]
\end{gathered}
$$

where $U$ is the horizontal wind speed, $w$ is the vertical wind speed, $T$ is the air temperature, $x$ is any scalar (e.g. the the $\mathrm{CO}_{2}$ or any other GHG component concentration, or - specific humidity $q$, etc.), $m_{d} / m_{w}$ is the ratio of molecular weight of the dry air to that of water vapor, and $\rho_{d}, \rho_{w}$ and $\rho_{x}$ are the densities for dry air, water vapor, and $x$ respectively. The right hand side of $\overline{w^{\prime} x^{\prime}}$ has extra terms due to corrections in air density. See also other corrections [10,11].

Depending on the Reunolds number boundary layer, flux may be laminar or turbulent. A laminar boundary layer flux is one where the flow takes place in layers, i.e., each layer slides past the adjacent layers. This is in contrast to Turbulent Boundary Layers where there is an intense agitation mixing across several layers of it. The mixing is now not only microscopic but on a macroscopic scale. Portions of fluid may move across. Thus there is an intense exchange of mass, momentum and energy on a much bigger scale compared to a laminar boundary layer.
The turbulent fluxes are formed only at larger Reynolds numbers. The scale of mixing cannot be determined by molecular viscosity alone. Those calculating turbulent flow are based on what is called Turbulence Viscosity or Eddy Viscosity, which has no exactly expressed clear mechanism. It needs to be modeled. Several models have been developed for the purpose.

New approach to flux modeling in this article is based on modification of the as basic concept as material particle concept. We will call it material point of the second type meaning that traditional concept of the material point as a small body with the negligible size but with a given amount of mass will be called material point of the first type.

\section{New Concept of Material Point}

In the traditional introductions into eddy covariance method one of basic primary concepts is an idea of fluid parcel which "is a very small amount of fluid, identifiable throughout its dynamic history while moving with the fluid flow" (wikipedia). Other close ideas are material point, particle. We introduce new conception called material point of the second type. The difference of the new conception from the traditional conception of the material point (let us call it material point of the first type) is following. The point of the second type is not characterized by its mass but by density, its motion is a motion of the continuous media - with deformations and torsion. Hereby we have said goodbye to the old sophism "let us call material particle material point and will treat it as a point." Sorry, material point is bit of matter. Therefore it has inalienable ability to be oriented relative to other material objects. And calling it point should not 
eliminate this essential ability. Treating material pint as geometrical point is sophism. Description of material point has to include this new degree of freedom.

This revelation brings us to reconsideration of the flux. A new aspect of the new model is awareness on necessity of projecting velocity vector $V_{\alpha}$ to configuration space of radius-vectors $R^{\beta}$ to manipulate with velocities Cartesian coordinates:

$$
V_{\alpha}=H_{\alpha \beta} R^{\beta},
$$

because velocity vector is an object of the different (tangential) space. For the fundamental geometrical aspects of this statement see [12].

Here $H_{\alpha \beta}$ is advection-distortion-vortex tensor (affinor, as it is called in [12], operator), $R^{\beta}$ is radius-vector of the material point. Splitting tensor $H_{\alpha \beta}$ into three parts, responsible for expansion $\theta \delta_{\alpha \beta} / 3$, shear $\sigma_{\alpha \beta}$ and vorticity $\omega_{\alpha \beta}$ is a novel tool to describe turbulence in the boundary layer agro-meteorology accounting, new degrees of freedom of the particle modeled as a material point of the second type:

$$
H_{\alpha \beta}=\theta \delta_{\alpha \beta} / 3+\sigma_{\alpha \beta}-\omega_{\alpha \beta} .
$$

\section{GHG Flux Model Equations}

Hydrodynamic Euler equations split into

$$
\begin{aligned}
& \frac{d \theta}{d t}+\frac{1}{3} \theta^{2}+\sigma^{2}-\omega^{2}=-F_{\alpha \alpha} \\
& \sigma^{2}=\sigma_{\alpha \beta} \sigma_{\alpha \beta}, \omega^{2}=\omega_{\alpha \beta} \omega_{\alpha \beta}, \frac{d \rho}{d t}=-\rho \theta, \\
& \frac{d \sigma_{\alpha \beta}}{d t}+\frac{2}{3} \theta \sigma_{\alpha \beta}+\sigma_{\alpha \gamma} \sigma_{\gamma \beta}-\frac{1}{3} \delta_{\alpha \delta} \sigma^{2}+\omega_{\alpha \beta} \omega_{\gamma \beta} \\
& \quad+\frac{1}{3} \delta_{\alpha \delta} \omega^{2}=-F_{(\alpha \beta)}+\frac{1}{3} \delta_{\alpha \delta} F_{\gamma \gamma} \\
& \frac{d \omega_{\alpha \beta}}{d t}+\frac{2}{3} \theta \omega_{\alpha \beta}+\sigma_{\alpha \gamma} \omega_{\gamma \beta} \\
& +\sigma_{\alpha \gamma} \omega_{\gamma \beta}+\omega_{\alpha \gamma} \delta_{\gamma \beta}=-F_{[\alpha \beta]}
\end{aligned}
$$

Here $F_{\alpha \beta}$ is a tensor of the external forces gradient. Dynamics of the turbulent flux (3) is much more complex than of laminar flux and flux measurement methods need including turbulence-related terms.

\section{Advection-diffusion-reaction Equations}

For a more general description structure (e.g. eddies) formation we get equation of the multi-component reaction-diffusion type

$$
\frac{\partial x_{i}}{\partial t}=f_{i}\left(\left\{x_{j}\right\}\right)+\nabla_{k} D^{k}{ }_{j}(r) \nabla^{j} x_{i}
$$

Substances diffusion and transfer of thermal energy are described by the same class equations. This equation is extremely universal and can be applied in modeling the broad range of processes taking place in agroindustry and in its energetics. The most impressive new application is a thermochemical decomposition of organic material such us agro manure at elevated temperatures without the participation of oxygen (pyrolysis) and production of designed fuels. It goes without saying that migration and generating (reacting) of gases in soil described by this equation.

The geometries of the subsystems are also considered in the modeling of the growth kinetics as a crucial factor. New class of equations called advection-diffusion-reaction (http://arxiv.org/pdf/1210.4091v2.pdf) was derived as following

$$
\frac{\partial \rho_{i}}{\partial t}=\frac{1}{3} H_{i} \rho_{i}+f\left(\left\{\rho_{i}\right\}\right)+\nabla\left[D_{i}\left(\left\{\rho_{i}\right\}\right) \nabla \rho_{i}\right]
$$

This equation describes arbitrary amount of material components with densities $\rho_{i}$, parameters $H_{i}$-diagonal elements of matrix in (1) - are responsible for an advection change of density, and coefficients of the effective diffusion $D_{i}$, generalized and adopted when needed. It may also provide nonlinear evolution scenarios for evolution of the multi-component reacting media in the different systems such as agro-bio-geo fluxes. The nonlinear term $f\left(\left\{\rho_{i}\right\}\right)$ stands for reactions between the components. The fluxes in the layers are objects for matching requirements on the boundaries between the layers. These requirements are fulfilled by appropriate identification of the constants of integration.

One of the new features of the nonlinear dynamic processes described by given equation is the existence of the so called threshold effects. This means that we may expect emergence and ability to long existence of some eddies and grow some of them to scales intensities of tornado before getting destroyed up.

\section{Speed of Sound in Turbulent Flux}

Next aspect in using physical laws in the presence of turbulence is taking into account vorticity in Doppler Effect for measurement of speed of flux. Traditionally eddy covariance theory refers to standard Doppler Effect whereas it is based on consideration of the flux as turbulent. In self-consistent model the basic equation describing (ultra)sound needed to be one for turbulent flux, e.g. Blokhintsev- Howe equation $[13,14]$. In the adiabatic approximation the equation holds

$$
\begin{aligned}
& B=H+v^{2} / 2, \frac{D}{D t}=\frac{\partial}{\partial t}+v \nabla, L=\Omega \times v-T \nabla S \\
& \left\{\frac{D}{D t}\left(\frac{1}{c^{2}} \frac{D}{D t}\right)+\frac{1}{c^{2}} \frac{D v}{D t} \nabla-\nabla^{2}\right\} B=\operatorname{div} L-\frac{1}{c^{2}} \frac{D v}{D t} L,
\end{aligned}
$$

Here $H$ is enthalpy, $\Omega=$ rotv is vorticity, $S$ - entropy, $T$ - temperature, $v$ is flux speed, $c$ is sound speed, $t$ is time. Note that drag enthalpy $B$ is connected to sound pressure $p$ as $\partial p / \partial t=\rho D B / D t$ ( $\rho$ is mass density of the media). Blokhintsev- Howe equation is derived as a consequence of impulse and mass conservation as well as equation of the state of the ideal gas. LHS of it correspond to the transfer of the sound in the arbitrary nonhomogeneous flux and RHS characterizes the sources of the sound connected to character of the flux such as presence of vorticity and entropy gradient. As one can see, sound equation much more complicated than those 
traditionally used for deriving Doppler Effect in the frame of eddy covariance method.

\section{Conclusions}

Dynamics of the turbulent GHG flux on the boundary layer, as given mathematical model demonstrates, is much complicated in comparison with vortex-free motion. Therefore, eddy covariance method, as an existing broadly accepted successfully working instrument, can experience further development and can bring even more detailed models, accounting vorticity. Vorticity is the phenomenon changing radically many of the scenarios in nature when accounted $[9,10]$. Therefore we may expect that existing questions in boundary level flux modeling, such as energy balance problem, can be tackled using vortex.

\section{Acknowledgements}

This research was funded by Government of Russian Federation, Grant № 11.G34.31.0079.

\section{References}

[1] Vasenev I.I., Valentini R. Experience in Organization Green House Gases Monitoring, C- and N-stockpiles in the Anthropologically Changed Ecosystems in the European Part of Russia. Report on the 1st Annual Scientific-Organizational Conference, Laboratory for Agro-ecological Monitoring, Modeling and Forecasting in Ecosystems (LAMP), Russian State Agrarian University - Moscow Agricultural Academy named after K.A.Timiryazev. December 12, 2012.

[2] Landau L.D., Lifshitz E.M. (1987). Fluid Mechanics. Vol. 6 (2nd ed.). Butterworth-Heinemann.
[3] Burba G. Eddy Covariance Method for Scientific, Industrial, Agricultural and Regulatory Applications. A Field Book on Measuring Ecosystem Gas Exchange and Areal Emission Rates. ISBN 978-0-615-76827-4. LI-COR Biosciences, Lincoln, Nebraska, 332 pp.

[4] Aubinet, M., Vesala T., and Papale D. (Eds.), 2012. Eddy Covariance: A Practical Guide to Measurement and Data Analysis. Springer, Dordrecht, London, New York, 442 pp.

[5] Nurgaliev I.S. «Singularities Are Averted by Vortices». Gravitation and Cosmology, 2010, Vol. 16, No. 4, pp. 313-315.

[6] Nurgaliev I.S.. Modern astronomical knowledge as component of general education for sustainable development. Astronomical and Astrophysical Transactions (AApTr), 2012, Vol. 27, Issue 3, pp. 429-430. ISSN 1055-6796, Cambridge Scientific Publishers.

[7] Nurgaliev I.S. "Confirmation of Cosmological Bounces Predicted by Alexander Friedmann." International Journal of. Modern Physics: Conference Series, Vol. 3 (2011), pp 281-285.

[8] Baldocchi D.D. “Assessing the eddy covariance technique for evaluating carbon dioxide exchange rates of ecosystems: past, present and future. Global Change Biology, Volume 9, Issue 4, pages 479-492, April 2003.

[9] .Webb, E. K., Pearman, G. I., and Leuning, R.: 1980, 'Correction of Flux Measurements for Density Effects Due to Heat and Water Vapour Transfer', Quart. J. Roy. Meteorol. Soc. 106, 85-100.

[10] Wyngaard J.C. 1990: Scalar fluxes in the planetary boundary layer -- Theory, modeling, Boundary-Layer Meteorology and measurement. Boundary Layer Meteorology, 50, 49-75.

[11] Fuehrer P. L., C. A. Friehe. Flux Corrections Revisited. BoundaryLayer Meteorology, 102: 415-457, 2002.

[12] Rashevsky P.K. Riemann Geometry and Tensor Analysis (in Russian), Nauka. Eds, Moscow, 1967.

[13] Blokhintsev D.I., Acoustics of a Non-homogeneous Moving Medium, Gostekhizdat, 1945 (English translation: N.A.C.A. Technical Memorandum no. 1399 (1956)).

[14] Howe D.I. Acoustics of Fluid-Structure Interactions, Cambridge University Press, Cambridge, 1998.

[15] Nurgaliev I.S. Physical Kinetics of Demography. Economic Strategies. - 2009. - N 1. - pp. 170-175.

[16] Nurgaliev I.S. Turbulency of the New Risks Demand Revising Strategies of Development. Economic Strategies - 2011, N 6, pp. 56-60. 